\title{
APUNTES SOBRE ALGUNAS LATIFOLIADAS DE MADERAS VALIOSAS 1. Nogal común (Juglans regia L.)
}

Verónica Loewe M. Ing. Forestal, División Silvicultura, INSTITUTO FORESTAL. Huérfanos 554, Santiago, Chile.

\section{INTRODUCCION}

El nogal común presenta actualmente gran interés en algunos países europeos, por ser uno de los protagonistas de la conversión productiva puesta en marcha en muchos terrenos ex-agrícolas, a causa de los excedentes de determinados productos en la Comunidad Economica Europea (Annali, 1987; Mercurio, 1988).

Es una especie de crecimiento relativamente rápido, al contrario de lo que se piensa habitualmente (Del Sole, 1983; Luna, 1990), que podría ser establecida en parcelas abandonadas o en aquellas que no son rentables para otros cultivos, al borde de caminos, en perímetros de cultivos, en fajas cortavientos, etcétera, alternativas que pueden ser idóneas para establecer plantaciones orientadas a la producción de madera, o a la producción combinada de madera y frutos (IDF, 1983).

Es de interés saber que actualmente la madera de nogal es una de las más cotizadas en el mercado europeo, con precios que oscilan entre los US $\$ 1.000$ y $4.000 / \mathrm{m} 3$ en pie, dependiendo de la calidad de la troza (Guglini, 1988). Esto evidencia la potencialidad de un recurso que, por lo demás, puede ser aprovechado desde el punto de vista frutícola aplicando las técnicas para ello adecuadas.

\section{ANTECEDENTES GENERALES}

\section{Descripción General}

La hipótesis más probable es que sea originado de China, o del macizo del Himalaya, y que desde allí haya pasado a la India, Persia, Grecia e Italia, desde donde habría sido difundido en la antiguedad a los otros países europeos (Forte, 1982).

Los escritos de Virgilio y de Ovidio afirman que ya durante la civilización griega era una planta doméstica y que en la época del Imperio Romano se la cultivaba ampliamente (Guglini, 1988).

El Juglans regia $\boldsymbol{L}$. es la especie más difundida y conocida de la familia Juglandaceae. Es un árbol vigoroso, de tronco solido, alto, recto, que se abre en una copa 
frondosa, amplia y armoniosa. Las hojas son compuestas, caducas, de un color verde intenso, olor bálsamico y sabor amargo astringente.

Es una especie monoica. Las flores masculinas, reunidas en amentos péndulos, aparecen sobre las ramas del año precedente antes de la aparicion de las hojas. Las flores femeninas son solitarias o reunidas en grupos de 2-3 y aparecen sobre los brotes nuevos del año, simultaneamente con las hojas. El fruto es una drupa de forma globosa. La semilla, rica en aceites, está dividida en 4 lóbulos rugosos e irregulares y madura en Abril - Mayo (Guglini, 1988).

El tronco posee una corteza lisa, la madera es compacta y con un veteado hermoso. El aparato radical es pivotante y las raíces tienden a colonizar las capas superficiales del suelo (IDF, 1983).

Es una especie de interés tanto agrícola como forestal, ya que produce frutos comestibles muy apreciados y madera de un valor considerable para la producción de muebles de estilo (tanto macizos como enchapados) y objetos artesanales (Bagnaresi, 1986). Actualmente es la madera más cotizada en el mercado europeo, lo que determinar un notable interés por parte de muchos propietarios por la posibilidad de combinar, con técnicas racionales, la producción frutícola con la producción de madera al final de la rotación, como se ha efectuado desde hace siglos en algunas zonas (Minotta, 1990).

\section{Exigencias Ecológicas.}

Es una especie de plena luz, muy sensible a la competencia de las malezas durante sus primeros años, siendo exigente en espacio vital (Denci et al., 1982; IDF, 1983), por lo que en las plantaciones frutícolas se usan espaciamientos superiores a los $10 \mathrm{~m}$. En parte debido a esta exigencia el nogal no tiene la reputación de ser un árbol forestal, mas aún siendo a menudo cultivado por sus frutos en las zonas agrícolas.

Con respecto a la exposición es un árbol que prospera bien en las laderas de mayor insolación; a este respecto sus exigencias son importantes, sobretodo para la producción de frutos, y algo menos para la producción de madera. Por esto conviene evitar tanto las exposiciones Sur como las exposiciones Norte, si el suelo no es capaz de asegurar una buena reserva de agua (IDF, 1983).

Es afectado tanto por el frío como por el calor excesivos, así como por las neblinas, la humedad y las heladas tardías (Forte, 1982). Se estima que necesita, al menos durante 6 meses, una temperatura media superior a los $10^{\circ} \mathrm{C}$ (IDF, 1983).

Se desarrolla bien tanto en planicies como en colinas, hasta los $800-1000 \mathrm{msnm}$ si las exposiciones le son favorables (Bagnaresi y Minotta, 1989; Denci et al., 1982). 
Crece perfectamente bien en pendiente, siempre que encuentre suelos profundos y frescos (por ejemplo en laderas cóncavas).

Las heladas bruscas después de un período templado pueden llegar a matar los nogales, pero si el frío llega gradualmente puede soportar hasta - $30^{\circ} \mathrm{C}$. Es una especie sensible a los vientos marinos (IDF, 1983).

Para un buen desarrollo el nogal necesita cerca de $700 \mathrm{~mm}$ de agua repartidos durante el año (Denci et al., 1982; Mezzalira, 1989). Tolera la humedad, pero prefiere los sitios donde en verano el aire es seco y el suelo permanece fresco. Es una especie sensible tanto a la aridez como a la escesiva acumulación de agua, ya que en estos últimos terrenos la aparición de Phytophtora y Armillaria pueden provocar la muerte de las plantaciones en pocos años.

Prefiere terrenos profundos (al menos $50 \mathrm{~cm}$ ), permeables y frescos. Las napas freáticas deben ser profundas, $0.8 \mathrm{~m}$ como mínimo, y su nivel poco variable (Bagnaresi et al., 1986; Denci et al., 1982).

Se puede desarrollar en suelos muy variados, siendo poco sensible a variaciones de la composición, pero siempre que no sean muy ácidos. El pH optimo se sitúa entre 6.5 y 7.5, y no hay que plantarlo si es inferior a 6.0 (IDF, 1983). Con valores superiores a 8.0 pueden presentarse síntomas de clorosis (Minotta, 1989). Acepta los terrenos arenosos siempre que posean una fuente de agua, que la arena no sea completamente estéril y que haya una vegetación acompanante que enriquezca en humus (IDF, 1983).

Es una especie exigente en sales minerales, particularmente nitrógeno, cuando es cultivada para obtener frutos, aunque para la producción de madera esto es menos evidente. En general crece bien donde la vid y el maíz dan buenas cosechas.

Estas exigencias de sitio obstaculizan su cultivo en superficies extensas, especialmente en las áreas de colinas y montañas, donde la naturaleza del terreno es variable en pocas decenas de metros. Pero, considerando el alto valor de su madera, es conveniente cultivarla en pequeñas superficies o en hileras a lo largo de caminos o alrededor de otros cultivos (Denci et al., 1982; IDF, 1983).

Es importante evitar terrenos muy compactos o susceptibles a inundaciones, así como los fondos de valles expuestos a las heladas tardías de primavera, dañinas para el desarrollo regular de la especie (Bagnaresi y Minotta, 1989).

\section{Principales Plagas y Enfermedades.}

Fuera de los grandes fríos y de las heladas repentinas, sus principales enemigos 
son los hongos Armillaria mellea, Phytophtora cactorum y Rosellinia necatrix, que provocan la muerte a través de la destrucción de las raíces. Lamentablemente no se conocen tratamientos contra estas enfermedades (Denci et al., 1982; IDF, 1983). También es sensible a la pudrición amarilla del tronco provocada por Inonotus hispidus.

Con respectos a los insectos, la Carpocapsa pomonella produce daños significativos a los frutos y esporádicamente también se pueden tener problemas con Cossus cossus y Zeuzera pyrina, lepidópteros que excavan galerías en la madera.

Es conveniente proteger las plantas de los ataques de conejos, liebres y otros mamiferos.

\section{SILVICULTURA.}

\section{Establecimiento de Plantaciones}

El establecimiento de una plantación se puede efectuar mediante una siembra directa, o mejor aún, usando plantas bien formadas, sanas y vigorosas (Denci et al., 1982).

Siembra Directa.

Es una técnica factible en los sitios no sujetos a heladas de primavera y tiene la ventaja de evitar los traumas de plantación, pero implica mucho trabajo, ya que las semillas y las plantas deben ser protegidas tanto de los animales como de la vegetación competidora (Del Sole, 1983). Para evitar daños por eventuales heladas de primavera se recomienda el empleo de procedencias que presenten brotación tardía.

\section{- Conservación de las Nueces.}

Las nueces se deben cosechar cuando empiezan a caer, ya que entonces están bien maduras, y se conservan hasta el invierno en un local aireado a temperatura ambiente. Pueden ser secadas levemente sin inconveniente (IDF, 1983).

Si la germinación es irregular en el tiempo, las operaciones culturales se complican notablemente. Por esto es preferible preparar las nueces antes, mediante una estratificacion, y sembrar solamente aquellas que empiezan a germinar. Con este objetivo, a fines de invierno las nueces pueden ser estratificadas, poniéndolas en capas de $4-5 \mathrm{~cm}$ alternadas con capas de arena del mismo espesor, donde permanecen hasta su germinacion en la primavera. Otra forma es disponerlas en una sola capa, reduciendo las manipulaciones y facilitando la observación del momento inicial de la germinación. 
- Siembra.

El terreno debe ser preparado igual que para una plantacion. Las nueces que empiezan a germinar se siembran a fines de septiembre - principios de octubre, colocándolas de modo que la sutura quede en posición vertical (IDF, 1983; Luna, 1990), y cubriéndolas con una capa del doble de su espesor. Es conveniente sembrar dos nueces juntas para elegir después de un año las plantas más vigorosas y evitar pérdidas.

Si no se ha efectuado una estratificación las nueces se siembran en otoño, aunque este sistema es menos seguro. Sin embargo, en las zonas donde abundan roedores y aves es preferible efectuar la siembra en primavera, cuando además ya haya pasado el peligro de las heladas tardías (IDF, 1983).

De todas maneras se recomienda la instalación de protecciones contra roedores y aves para evitar pérdidas que pueden llegar a ser considerables (Bagnaresi y Minotta, 1989). Para esto puede ser útil la instalación de conos de malla de unos $15 \mathrm{~cm}$ de altura, parcialmente enterrados, así como también de trampas. También es importante eliminar las malezas, siendo una buena solución para ello el mulching realizado en plástico, que se puede instalar simultaneamente con la siembra.

Plantación.

En general es preferible la plantación debido a que las plantas ya desarrolladas resisten más fácilmente a las heladas, a las cuales el nogal es muy sensible (Guglini, 1988).

- Características de las Plantas.

En una plantación bien cuidada, hecha con plantas cuyas raíces no han sido daffadas, la recuperacion del nogal es buena. Se pueden emplear sin mayores problemas plantas tanto pequeñas como grandes, de 1 a 4 años, con una altura entre 0.5 y $2.5 \mathrm{~m}$ (Bagnaresi y Minotta, 1989; Luna, 1990).

Se deben emplear plantas vigorosas, derechas, sin deformaciones ni daños apicales y con un sistema radicular bien conformado (Denci et al., 1982).

El trasplante se efectúa en maceta, evitándo las corrientes de aire, el sol directo y una atmósfera desecante, y efectuando la plantación lo antes posible.

- Densidad de Plantación.

El espaciamiento depende del objetivo de produccion. En plantaciones con 
finalidad mixta se necesitarán distanciamientos mayores que aquellos necesarios para la producción de madera.

Los franceses recomiendan la realización de plantaciones con una densidad igual a la densidad de cosecha, lo que corresponde a un distanciamiento de $10 \times 10$ a $12 \times 12$ $\mathrm{m}$. Este tipo de plantaciones a baja densidad debe ser efectuado solamente con material seleccionado y las operaciones culturales se deben realizar en forma individual (IDF, 1983).

En plantaciones de alta densidad queda la posibilidad de hacer una selección posterior y además hay otras ventajas implicitas, como que las operaciones culturales asumen una importancia relativa menor, el crecimiento en altura se ve favorecido durante los primeros años, la poda se ve facilitada y los árboles están un poco más protegidos de las heladas (IDF, 1983; Nicholas, 1988).

Otra formula propuesta consiste en plantar el doble del número de plantas que se desea cosechar para elegir las mejores, lo que es importante siendo las plantas genéticamente heterogeneas.

En todo caso no existen indicaciones claras respecto a la densidad ideal de plantaciones para produccion de madera y se citan distanciamientos desde $5 \times 5$ hasta 3 x 3 m (Bagnaresi et al., 1986; Denci et al., 1982).

En Italia, en base a las experiencias del Istituto Sperimentale per la Selvicoltura di Arezzo, recomiendan la instalación de plantaciones a alta densidad, de $625-1111$ plantas / ha, con un distanciamiento tal que permita la mecanizacion de las operaciones culturales (Mercurio, com. personal).

En base a la falta de experiencia en el país, a las características del material con que se cuenta y a los antecedentes mencionados, se recomienda el establecimento de plantaciones de nogal a alta densidad ( $3 \times 3$ a $4 \times 4 \mathrm{~m}$ ), siempre que se efectúen oportunamente raleos, en cuanto las copas de los árboles empiecen a tocarse.

- Preparación del Terreno.

El ideal es arar o subsolar todo el terreno (Bagnaresi et al., 1986), pero si esto no es posible, se tratarán de hacer casillas lo suficientemente grandes como para favorecer un correcto establecimiento y recuperación de las plantas (Guglini, 1988). Estas últimas se pueden abrir manualmente, en forma mecanizada o con un taladro, aunque en los suelos limosos o mas o menos arcillosos este último tiene el inconveniente de alisar y sellar las paredes de la casilla. Para evitar este inconveniente conviene abrir las casillas en otoño y plantar más tarde, solo en primavera (IDF, 1983). 


\section{- Plantación.}

Se realiza desde fines de mayo o inicios de junio hasta la primavera en el caso de suelos más pesados (casillas abiertas con anticipación). En primavera se debe plantar lo más tarde posible, justo antes de la brotación, sobretodo en los terrenos desnudos y expuestos al viento, por el riesgo de heladas tardías.

Las raíces heridas o necrosadas deben ser eliminadas para reducir el riesgo de pudrición y para favorecer la emisión de nuevas raíces.

Se debe cuidar de no enterrar el cuello de la planta, porque esto condiciona un mal desarrollo de la misma. Por el contrario, conviene poner las plantas a un nivel un poco más alto del que tenían en el vivero, ya que se ha visto que si se cubre el cuello $5-10 \mathrm{~cm}$ se produce un atraso del desarrollo de alrededor de 3 - 4 años (IDF, 1983).

Es indispensable en las zonas donde hay conejos y liebres, utilizar protecciones como mallas metálicas, Corromet u otras.

La instalación de tutores puede ayudar a corregir individuos mal formados y a favorecer un correcto desarrollo de la planta durante sus primeros años. Estos deben ser colocados antes que las plantas, enterrando una vara en la casilla y amarrando después la planta a ella con un material elástico para evitar posibles roces (Guglini, 1988). Es mejor situarlos en la dirección de los vientos dominantes. En general se tratarán de omitir si no se hacen realmente indispensables (IDF, 1983; Luna, 1990).

- Enmiendas y Fertilización.

Una enmienda del terreno es aconsejable en suelos de texturas extremas. Es posible utilizar humus con el objeto de mejorar la estructura y aumentar la retención de humedad, estimulandose así el crecimiento de las plantas.

Se tiene poca información acerca de la respuesta del cultivo a la fertilización. El nogal es poco exigente en ácido fosfórico y en potasio, pero al parecer al menos durante los primeros años es oportuna la distribucion, a fines de invierno, de un abono ternario con alto tenor de Nitrógeno, del orden de $1-2 \mathrm{Kg}$ / planta (Bagnaresi et al., 1986; Denci et al., 1982). Otros autores recomiendan $100 \mathrm{gr} \mathrm{N} /$ árbol / año de edad, distribuidos en 2 aplicaciones (Minotta, 1990).

La fertilización debe ir acompañada de una remoción superficial del suelo, o de un mulch, ya que de lo contrario puede favorecer a la vegetación competidora más que a los árboles mismos. Esta es una técnica muy beneficiosa, ya que mantiene la frescura, 
favorece la acción de las lombrices y por lo tanto la aireación del suelo, e impide el desarrollo de malezas (principalmente gramíneas) alrededor de las plantas. Se debe hacer en primavera, para que en invierno el material vegetal esté descompuesto. Puede ser hecho ya sea con un plástico negro, o con una capa de carbón o material vegetal (corteza u otro) (IDF, 1983).

\section{Téenicas Culturales.}

Son indispensables, ya que el objetivo final es producir un fuste de buena calidad, sin nudos ni defectos y de una dimensión determinada.

Deben ser efectuadas individualmente, especialmente en el caso de plantaciones a baja densidad, ya que perder un árbol tiene un costo muy alto. Son frecuentes durante los primeros años después de la plantación, pudiéndose dar por terminada después de 12 años, según la fertilidad del sitio y la especie empleada (IDF, 1983). En el caso de plantaciones a alta densidad, estas prácticas culturales continuan posteriormente con los raleos.

\section{- Control de Competencia.}

Durante los primeros affos se recomienda mantener el suelo limpio alrededor de las plantas, ya que las malezas constituyen el gran enemigo de los nogales. Con este fin se puede elegir entre una remoción manual o mecanizada, un mulching, o la aplicación de tratamientos químicos (Bagnaresi et al., 1986).

\section{- Cultivos Intercalados.}

Las operaciones que se deben procurar durante los primeros 3 - 4 años, que son las más costosas, se puedan compensar efectuando asociaciones apropiadas con otras especies acompañantes. Estas pueden ser arbustivas o arboreas, coniferas o latifoliadas, pero deben proporcionar, en rotaciones cortas, productos de valor comercial. Estos pueden estar representados por postes, leña y otros productos menores. También es posible incorporar cultivos herbáceos (forraje, plantas medicinales, flores para la apicultura, etcétera). Esta vegetación acompañante puede mejorar la forma de los árboles y facilitar la formación del fuste (Annali, 1987). Con este objetivo en el sur de Francia han uitilzado el Elaeagnus angustifolia (Olivo de Bohemia), que se siembra fácilmente y que fija nitrogeno atmosférico.

Para reducir el riesgo de daf́os mecánicos a los troncos y la competencia ejercida por dichos cultivos, es aconsejable dejar una faja no cultivada alrededor de los árboles (Forte, 1982). No se se debe cultivar alfalfa conjuntamente con nogales, ya que ésta 
produce un efecto inhibitorio del crecimiento, y tampoco conviene abusar de los cereales (trigo, cebada, centeno, avena); en cambio el maíz da buenos resultados (IDF, 1983).

Estos cultivos intercalados son interesantes desde el punto de vista económico, ya que permiten obtener ingresos a partir del suelo no utilizado durante los primeros años de vegetación de los nogales, cuando estos cubren solamente una pequeña parte del espacio disponible, pero se debe evitar que estos cultivos anexos compitan con los nogales.

- Podas de Formacción.

Son necesarias para corregir las bifurcaciones del fuste, para sustituir las flechas dañadas por factores bióticos o abióticos y para eliminar las ramas más gruesas y vigorosas que puedan competir con la flecha principal. Si se planea una producción principalmente de madera, se harán necesarias podas adicionales para asegurar un fuste libre de nudos en la parte baja.

Formación del Brote Principal:

La formación de la flecha es necesaria cuando el ápice ha sido destruido, o cuando crece demasiado poco, así como cuando hay varios ápices que compiten entre sí.

El objetivo de esta operación es el favorecer el desarrollo de una flecha única, vigorosa y recta. Para ello es preferible intervenir cuando los ápices todavía estan verdes, al inicio del periodo vegetativo.

Si las ramas aún están verdes, se procede de la siguiente forma:

Si el ápice ha sido destruido o si es poco vigoroso, la flecha se reconstituye a partir de una rama, para lo cual no necesariamente se debe elegir la rama más cercana al ápice, ya que una rama inferior vigorosa y lo suficientemente vertical dará igualmente un buen resultado. Si la rama es prácticamente vertical se elimina el ápice original justo en el punto de inserción de la rama elegida. En cambio si la rama es oblicua se acerca al ápice por medio de un amarre, cortando el original por sobre la ligadura, y una vez que la rama se lignifique el muñon apical que sirve como tutor será eliminado.

Si ninguna de las ramas es apta para reemplazar al ápice, la solución es cortar la mayoría de las ramas situadas en la parte superior y en esta zona aparecerán entonces nuevas ramas verticales de entre las cuales se elegirá la mejor.

Si las ramas ya están lignificadas, se puede proceder de tres maneras: 
Poda de la flecha: Cuando entre las ramas de un árbol de mala forma se encuentra una que pueda llegar a constituirse como flecha principal, se recurre a una poda cuyo objetivo es dar a aquella rama una dominancia sobre las otras, las que son acortadas o eliminadas. Así, con el tiempo, el tronco inclinado se enderezará, proceso que se puede acelerar mediante la instalación de un tutor.

Enderezamiento por medio de ligaduras: Cuando no existe una flecha única, sino que varias lignificadas y demasiado inclinadas para esperar que se logren enderezar por sí solas, es posible conservar la más derecha uniéndola a otra rama y las ramas restantes se cortan por debajo del nivel del amarre.

Despunte: Cuando las soluciones anteriores no son practicables, hay que efectuar un despunte de la planta en primavera y de ese modo se renuevan una o varias ramas apicales de las cuales posteriormente se selecciona la mejor por medio de los procedimientos descritos anteriormente.

Control de Ramas Gruesas:

La poda de formación debe limitar el crecimiento de las ramas que se desarrollan mucho, o que tienen una tendencia a la verticalidad, cualquiera sea su nivel dentro de la copa.

Todas las ramas que se enderezan demasiado son potencialmente peligrosas y es necesario eliminarlas o acortarlas. En cambio las ramas horizontales se pueden conservar hasta que alcancen los $3-4 \mathrm{~cm}$ de diámetro.

Las ramas que se eliminen deben ser cortadas a ras del tronco, pero sin dañar el anillo situado en el nivel de inserción de la rama, denominado anillo cicatricial.

- Podas para obtener un Fuste Libre de Nudos.

Se puede esperar un fuste libre de nudos de $4 \mathrm{~m}$ de altura $\mathrm{y}$, en condiciones ambientales adversas, de no menos de $2.5-2.8 \mathrm{~m}$, ya que bajo estas dimensiones el precio del metro cúbrico se castiga (Luna, 1990).

Mediante estas podas se eliminan sistemáticamente las ramas presentes en la parte inferior del fuste, independientemente de su vigor, cuidando de no sobrepasar el tercio de la altura total.

Se inician despues que se ha realizado la poda de formación, nunca antes de los 2 - 3 años de edad, cuando los árboles sobrepasan $1.5 \mathrm{~m}$ de altura, o cuando las ramas 
presentan un diámetro de inserción en el tronco de entre $1-3 \mathrm{~cm}$. Las ramas más gruesar deben ser cortadas primero, cualquiera sea su localización en el tronco (IDF, 1983).

Los criterios a seguir para ejecutar bien las podas son (Bagnaresi y Minotta, 1989; Denci et al., 1982; IDF, 1983) los siguientes:

* Podar el tercio inferior del árbol.

* Podar todas las ramas situadas en el sector en que el tronco tiene entre 10 y 15 cm de diámetro.

* Podar todas las ramas cuya base mida entre 3 - 4 cm de diámetro, no importando su localización en el tronco.

Sin embargo, en base a los últimos datos experimentales obtenidos en Francia, se ha insistido en la necesidad de no podar más allá del 30\% del total de la copa del árbol, ya que de lo contrario se estaría alterando negativamente el desarrollo del mismo.

La frecuencia de las podas depende de la velocidad de crecimiento de los árboles, siendo tanto más frecuentes mientras más vigorosos son los individuos, y suspendiéndose cuando se alcanza la altura del fuste libre de nudos definida como objetivo.

Con respecto a la época de ejecución es la misma que para la poda de formación, es decir a fines de invierno. Se debe tratar de evitar podar durante periodos fríos, a continuación de los cuales se pueden observar exudaciones abundantes de savia (Denci et al., 1982).

Además, durante los primeros años en primavera se pueden eliminar las yemas epicormicas, operación manual fácilmente ejecutable y que prácticamente no deja cicatrices (Bagnaresi et al., 1986; Denci et al., 1982).

\section{Plantaciones con Finalidad Mixta.}

La producción frutícola tradicionalmente conocida puede ser fácilmente conciliada con la producción de madera.

Este tipo de plantaciones difiere de las plantaciones para la produccion de madera sobretodo con respecto a la elección del material a utilizar. Si se desea obtener una produccion frutícola de valor comercial es necesario recurrir al uso de plantas injertadas con las variedades seleccionadas por su fruto, eligiendo las más idoneas para el ambiente en el que se desea realizar la plantación. Para las zonas de colina y de montaffa pueden aconsejarse, mientras no se disponga de indicaciones experimentales más precisas, los 
cultivares franceses tardíos, como "Mayette", "Parisienne", "Corne" y "Franquette". En las cotas menos elevadas el CV californiano "Hartley", así como otras variedades autóctonas (Bagnaresi et al., 1986; Minotta, 1989; Minotta, 1990).

Una vez que se hayan elegido las variedades, que deberán cumplir con una serie de requisitos productivos y ecológicos, se procederá a realizar los injertos. Como es sabido, esta técnica afecta la calidad de la madera, inconveniente que puede ser paliado injertando las plantas a nivel del cuello, o sobre las ramas de árboles ya adultos (Minotta, 1990). En último término se pueden emplear plantas de semilla de ciertas poblaciones que producen nueces de calidad aceptable.

Al efectuar la plantación hay que distribuir en forma adecuada un 5 - $10 \%$ de polinizadores, localizándolos en la dirección del viento (Minotta, 1989).

En este caso las técnicas de cultivo se mantienen. La diferencia fundamental es que la copa deberá formarse a mayor altura respecto a lo tradicionalmente usado en las plantaciones para producción de nueces, y con respecto a la madera se obtendrán fustes libres de nudos de menor longitud (2.5-3.0 m) (Bagnaresi et al., 1986), pero con la ventaja de tener ingresos adicionales distribuidos en el tiempo durante gran parte de la rotación. Para obtener una planta con esas características se deberán realizar podas según las modalidades ya descritas.

Para asegurar una producción frutícola buena y constante son indispensables riesgos de emergencia si el regimen pluviometrico no satisface las exigencia hídricas del nogal durante la fase de crecimiento de los frutos (Minotta, 1989).

Se deben tratar de evitar daños al tronco, sobretodo en la primavera cuando la corteza se desprende fácilmente, así como la poda de ramas demasiado gruesas, que alteran la calidad de la madera.

Los distanciamientos aconsejados son del orden de 7 × 8,8 × 9 y $8 \times 10$ metros, y hasta los 10 x 10y 10 x 12 (Bagnaresi etal., 1986), lo que corresponde al distanciamiento definitivo, o en hileras, a 8 - $10 \mathrm{~m}$ (Minotta, 1989).

Para resguardar la produccion frutícola hay que hacer tratamientos antiparasitarios contra Carpocapsa pomonella, con insecticidas, y contra la bacteriosis o peste negra (Xanthomonas juglandis) y la antracnosis (Gnomonia leptostyla), con productos cúpri$\cos$. También es de gran utilidad hacer fertilizaciones a fines de invierno y durante la maduración de los frutos con abonos ternarios con un alto tenor de nitrógeno (Bagnaresi et al., 1986). 
Las plantaciones se podrán cosechar aproximadamente a los 40 - 50 años, dependiendo de la fertilidad del terreno y de las dimensiones de los productos que se desea obtener (Minotta, 1990).

\section{ASPECTOS PRODUCTIVOS.}

En buenas condiciones se ha visto que hasta los 60 - 70 años los nogales presentan un incremento anual de $0.8-1.0 \mathrm{~cm}$ de diámetro (Bagnaresi et al., 1986; Bagnaresi y Minotta, 1989), alcanzándose así volúmenes que poseen un valor superior al de otras especies de maderas nobles, como por ejemplo hayas y encinas. Según IDF (1983), si el terreno es suficientemente fértil, a los 50 - 60 años se puede obtener un DAP de 50 - 60 $\mathrm{cm}$.

Las rotaciones oscilan entre los 40 y 60 años (Bagnaresi et al., 1986; Bagnaresi y Minotta, 1989), según la fertilidad del sitio y las características de crecimiento de las procedencias empleadas. El número final de árboles por hectárea debe ser de unos 70 individuos, no sobrepasando los 100 .

Con respecto a la productividad, se estima que una plantación de nogales sometida a las técnicas adecuadas puede llegar a proporcionar 150 - $200 \mathrm{~m} 3$ / ha en 40 años. Esta productividad es muy inferior a la proporcionada por especies de rápido crecimiento, como por ejemplo alamo y pino, pero su importancia económica - financiera es muy superior. Por esto en una elección de ese tipo hay que salir de los esquemas tradicionales que tienden a evaluar las especies principalmente en función de la cantidad de madera extraible por unidad de tiempo.

\section{USO Y COMERCIALIZACION DE LA MADERA.}

La madera de nogal es muy apreciada. Se trata de una madera semi - dura (densidad de $0.63-0.75 \mathrm{gr} / \mathrm{cm} 3$ ), de grano fino, de fibra larga, homogenea, fácil de trabajar, estable y muy estética (Mezzalira, 1989). Sus características físicas y químicas, unidas a sus cualidades estéticas indiscutibles, la convierten en una de las más bellas y valiosas (Luna, 1990).

Los fustes de mayor diámetro se destinan al trozado y a la producción de chapas para su utilización en ebanistería. La madera de calidad pero de menores dimensiones se emplea en carpintería fina, en la fabricación de culatas de armas y para esculturas.

Como por lo general estos cultivos se efectuán en superficies pequefias debido a las exigencias ecológicas, se podría pensar que la comercialización es problemática. Sin 
embargo existen empresas que requieren modestas cantidades de materia prima, pues actúan a nivel artesanal, y en el caso de la gran industria esto se podría obviar a través de la constitución de asociaciones entre los productores.

Además de la madera, el nogal presenta múltiples usos para los frutos (Guglini, 1988). Las nueces pueden ser consumidas tanto frescas como transformadas en confitería, o aún verdes, confitadas o en licores. De los cultivares idoneos se extrae el aceite de nuez, comestible y con usos industriales.

Las hojas, ricas en taninos y aceites esenciales, son usadas por la industria farmaceútica y de colorantes. El epicarpio del fruto, también rico en taninos, se usa en curtiduría, en la industria de colorantes, y con finalidades farmaceúticas. Plinio narra que era usado para teñir tejidos y los cabellos.

El mesocarpio del fruto, leñoso, se puede usar como combustible, y sus cenizas constituyen un optimo abono fosfo - postásico.

Por último, y hasta hace no mucho tiempo, en algunos países europeos, en las zonas montañosas y de colina, cuando nacía una niña plantaban en el jardín un nogal que al momento de su matrimonio iba a constituir su dote.

\section{REFERENCIAS.}

Annali del l' Instituto Sperimentale por la Selvicoltura di Arezzo. 1987. Noce e Ciliego da legno: esane de la produzione vivaistica nell 'Appennino centro - settentrionale. Vol. XVIII. Pp. $177-213$.

Bagnaresi U. et al., 1986. Il miglioramento dei boschi e la possiblilita di recupero alla produzione legnosa dei terreni agricoli abbandonati nella collina italiana. In: Studio Generale della Colina Italiana. Ed. Edagricole. 386 p.

Bagnaresi U. y Minotta G. 1989. Difesa e Salvaguardia dell'Appennino Emiliano - Romagnolo. Pratiche di Selvicoltura. IRECOOP. Emilia - Romana. 72 pp.

Del Sole E. 1983. Il noce nostrano, preziosa specie per il ricupero di terreni marginali. Rev. E -M No 9 - 10. Pp 48 - 53.

Denci L.; Mercurio R.; Moroni M.; Tocci A. 1982. Le possibilità di coltivazione del noce da legno. Revista Agricoltura e Ricerca № 14. Pp. 36 - 41.

Forte V. 1982. Il Noce. Ed. Edagricole. 80 pp. 
Guglini M. 1988. Il noce. Revista Verde Foreste № 4. Pp 15 - 17.

INSTITUT POUR LE DEVELOPPEMENT FORESTIER. 1983. La culture des noyers a bois. $89 \mathrm{pp}$.

Luna L, F. 1990. El nogal. Producción de fruto y de madera. Ed Mundi - Prensa. 155 pp.

Mercurio R. 1988. Il noce da legno nell 'Italia centro - settentrionale. Rev. Cellulosa e Carta № 2. Pp. $24-33$

Mezzalira G. 1989. Biologia e Ecologia delle principali specie da impiegare nell 'Arboricoltura da legno. Rev. Le Foreste № 5.

Minotta G. 1989. La coltura del noce da frutto ed a duplice attitudine produttiva in Italia. Revista Frutticoltura № 7. Pp 23 - 29.

Minotta G. 1990. La coltura del noce da frutto ed a duplice attitudine productiva in colina ed in montagna. Rev. Monti e Boschi № 1. Pp. 27 - 33.

Nicholas I.D. 1988. The silviculture of black walnut in New Zealand. In: Proceedings of papers contributed and/or presented and histories of Australian forestry and forest products, Institutions and Associations. 25 April - 1st May 1988- Albury - Wadonga. Vol. IV. 